\title{
An Insight Into Cervical Cancer Screening and Treatment Capacity in Sub Saharan Africa
}

\author{
Jenell S. Coleman, MD, MPH, ${ }^{1}$ Michelle S. Cespedes, MD, MS, ${ }^{2}$ Susan Cu-Uvin, MD, ${ }^{3}$ \\ Rose J. Kosgei, MBChB, MMED, MSc, ${ }^{4}$ May Maloba, ${ }^{5}$ Jean Anderson, MD ${ }^{1}$ Timothy Wilkin, MD, ${ }^{6}$ \\ Antoine Jaquet, $\mathrm{MD}, \mathrm{PhD},{ }^{7,8}$ Julia Bohlius, $\mathrm{MD}, \mathrm{MScPH},{ }^{9}$ \\ Kathryn Anastos, $M D,{ }^{10}$ and Kara Wools-Kaloustian, $M D^{11}$
}

\begin{abstract}
Objective: Approximately $85 \%$ of cervical cancer cases and deaths occur in resource-constrained countries where best practices for prevention, particularly for women with HIV infection, still need to be developed. The aim of this study was to assess cervical cancer prevention capacity in select HIV clinics located in resource-constrained countries.

Materials and Methods: A cross-sectional survey of sub-Saharan African sites of 4 National Institutes of Health-funded HIV/AIDS networks was conducted. Sites were surveyed on the availability of cervical cancer screening and treatment among women with HIV infection and without HIV infection. Descriptive statistics and $\chi^{2}$ or Fisher exact test were used as appropriate.

Results: Fifty-one (65\%) of 78 sites responded. Access to cervical cancer screening was reported by 49 sites $(96 \%)$. Of these sites, 39 (80\%) performed screening on-site. Central African sites were less likely to have screening on-site $(p=.02)$ versus other areas. Visual inspection with acetic acid and Pap testing were the most commonly available on-site screening methods at $31(79 \%)$ and $26(67 \%)$ sites, respectively. High-risk HPV testing was available at $29 \%$ of sites with visual inspection with acetic acid
\end{abstract}

${ }^{1}$ Department of Gynecology and Obstetrics, Johns Hopkins University School of Medicine, Baltimore, MD; ${ }^{2}$ Division of Infectious Diseases, Department of Medicine, Icahn School of Medicine at Mount Sinai, New York, NY; ${ }^{3}$ The Warren Alpert Medical School of Brown University, Providence, RI; ${ }^{4}$ Department of Obstetrics and Gynecology, University of Nairobi; ${ }^{5}$ Kenya Medical Research Institute FACES, Nairobi, Kenya; ${ }^{6}$ Division of Infectious Diseases, Weill Cornell Medical College, New York, NY; ${ }^{7}$ Université Bordeaux and ${ }^{8}$ INSERM, ISPED, Centre INSERM U897-Epidémiologie-Bio statistique, Bordeaux, France; ${ }^{9}$ Institute of Social and Preventive Medicine, University of Bern, Bern, Switzerland; ${ }^{10}$ Department of Medicine, Montefiore Medical Center, Bronx, NY; and ${ }^{11}$ Division of Infectious Diseases, Indiana University School of Medicine, Indianapolis, IN

Correspondence to: Jenell S. Coleman, MD, MPH, Johns Hopkins University School of Medicine, Phipps Bldg, Room 249, 600 N Wolfe St, Baltimore, MD 21287. E-mail: colemanj@jhmi.edu

The authors have declared they have no conflicts of interest.

J.S.C. was supported in part by a 2014 developmental grant from the Johns Hopkins University Center for AIDS Research (CFAR), an National Institutes of Health (NIH) funded program (P30AI094189), which is supported by the following $\mathrm{NIH} \mathrm{Co-Funding} \mathrm{and} \mathrm{Participating} \mathrm{Institutes}$ and Centers: NIAID, National Cancer Institute (NCI), NICHD, NHLBI, NIDA, NIMH, NIA, FIC, NIGMS, NIDDK, and OAR. K.W. was supported by NIH grant U01AI069911, which is supported by NIAID, NCI, NICHD S.C.U. was supported by the Lifespan/Tufts/Brown CFAR (P30AI42853) and The Miriam Hospital AIDS Clinical Trials Group (U10AI069472).

M.S.C. was supported in part by NIH grant UM1 A1068636. Support was also obtained in part by University of Washington CFAR 5P30AI027757; Case Western Reserve University CFAR 5P30AI036219; University of North Carolina Chapel Hill CFAR 5P30AI050410; University of California San Francisco CFAR 5P30AI027763; Harvard Medical School CFAR P30AI060354; and Albert Einstein College of Medicine CFAR 5P30AI051519. The International Epidemiologic Databases to Evaluate AIDS West and East Africa collaboration was funded by NCI, the Eunice Kennedy Shriver National Institute of Child Health and Human

Development (NICHD), and the National Institute of Allergy and Infectious

Diseases (NIAID) of the US NIH, as part of the IeDEA under Award

Number U01 AI069919 and U01AI069911, respectively.

(C) 2015, American Society for Colposcopy and Cervical Pathology and $50 \%$ of sites with Pap testing. Cryotherapy and radical hysterectomy were the most commonly available on-site treatment methods for premalignant and malignant lesions at $29(74 \%)$ and $18(46 \%)$ sites, respectively.

Conclusions: Despite limited resources, most sites surveyed had the capacity to perform cervical cancer screening and treatment. The existing infrastructure of HIV clinical and research sites may provide the ideal framework for scale-up of cervical cancer prevention in resource-constrained countries with a high burden of cervical dysplasia.

Key Words: HIV, cervical cancer, Pap, HPV, VIA

( Lower Gen Tract Dis 2016;20: 31-37)

ervical cancer is the fourth most common cancer in women worldwide, and in 2012, there were 528,000 new cases and 266,000 deaths. ${ }^{1}$ An estimated $85 \%$ of new cases and almost 9 of 10 deaths from cervical cancer occur in resource-constrained regions. $^{2}$ In sub-Saharan Africa (SSA), there are more than 250 million women who are at risk of developing invasive cervical cancer (ICC). ${ }^{1}$ The annual incidence of ICC is 50 per 100,000 women in SSA, and in 2012, there were more than 75,000 new cases reported and more than 50,000 women died from the disease. ${ }^{1,3}$

Cervical cancer can be prevented through comprehensive cervical cancer screening and treatment programs. According to the National Cancer Institute, the annual incidence of ICC in the United States decreased by $80 \%$ after Pap testing was widely adopted. ${ }^{4}$ Conversely, the incidence of ICC is expected to rise in SSA for the next 20 years because of lack of appropriate cervical cancer prevention services, including high-risk human papillomavirus (hrHPV) vaccination programs, the projected doubling of the population, and the disproportionate burden of HIV in the region. ${ }^{1}$ Studies have consistently shown that women with HIV infection have higher prevalence and longer persistence of oncogenic hrHPV subtypes than women without HIV infections. ${ }^{5-7}$ Because SSA has the highest burden of HIV infection among women, who are living longer because of the success of HIV treatment, a perfect storm has been created that is anticipated to increase the incidence of ICC. ${ }^{8}$ However, effective and feasible screening and treatment approaches need to be developed among women with HIV infection in this region.

Although Pap testing has been recommended for routine cervical cancer screening in the United States for more than 50 years, in resource-constrained settings, it is not feasible because of high cost and the need for cytology services, well trained experienced personnel, internal and external control mechanisms, and multiple clinic visits for the patients. Some screening guidelines have incorporated hrHPV testing (using either molecular biomarkers such as DNA and RNA or oncoproteins) into the screening process, which has shown high sensitivity. ${ }^{9-11}$ Although the cost of hrHPV testing is decreasing, it may still be too costly in some settings. As a result, many resource-constrained settings have focused on service delivery models using alternatives to the Pap test 
and hrHPV testing, such as visual inspection with acetic acid (VIA) or Lugol solution (VILI) with same-day cryotherapy in the see-and-treat approach. Although there is some controversy around the impact of VIA on reducing ICC incidence and mortality, it is a cost-effective and practical tool that has been embraced by the World Health Organization for implementation in resourceconstrained settings. ${ }^{12-15}$

Furthermore, treatment algorithms for cervical dysplasia and cancer vary from region to region and the management of ICC is a major challenge due to the lack of treatment options. Recently, there have been discussions about the potential to expand existing cervical cancer prevention programs; however, the current infrastructure is not well defined. ${ }^{16}$ The aim of this study is to describe the current capacity of cervical cancer prevention programs affiliated with NIH-funded HIV/AIDS networks that are located in SSA in anticipation of the need-based expansion of these programs.

\section{METHODS}

This was a cross-sectional survey of SSA sites affiliated with the following 4 NIH-funded HIV/AIDS networks: Centers for AIDS Research (CFAR), International Epidemiologic Databases to Evaluate AIDS (IeDEA), AIDS Clinical Trials Group (ACTG), and the AIDS Malignancy Consortium (AMC). However, because all responding AMC sites were also ACTG sites, for the purpose of this analysis, they are reported as ACTG sites. Other sites with more than 1 affiliation were categorized on the basis of the network that they listed on the survey. Although CFAR, ACTG, and AMC are large innovative HIV/AIDS research networks that support clinical trials, the IeDEA network collects data generated during the course of routine care within HIV clinics and uses these data to answer operational and outcomes questions related to antiretroviral rollout. The survey included questions about availability of cervical cancer screening, either on-site or through referrals, availability of treatment for premalignant and malignant lesions, and available personnel (see Table 1). Respondents were asked to describe their site and were able to select more than 1 option. Surveys were emailed to the affiliated sites and completed by a site representative between February and December 2013. For the IeDEA sites, this study was considered part of the parent study, which received approval from the institutional review boards previously. For the other sites, the study was exempt from institutional review board review because it was considered nonhuman subjects research.

\section{Statistical Methods}

The proportion of sites with screening and/or treatment, personnel, and methods of screening and/or treatment were calculated. Descriptive and comparative statistics were performed using SPSS v.22.0. The Pearson $\chi^{2}$ test or Fisher exact test was used for categorical variables, as appropriate.

\section{RESULTS}

Fifty-one $(65 \%)$ of 78 sites that were invited to participate completed the survey (see Figure 1). Among the 27 nonresponding sites, $19(70.4 \%)$ were colocated in the same country as 1 or more responding sites with $16(59 \%)$ colocated in the same city. Only $8(29.6 \%)$ of the nonresponding sites, representing 6 countries (Benin, Mali, Ethiopia, Ghana, Congo, and Mozambique), were located in countries from which no responses were received. Most respondents were represented by IeDEAaffiliated HIV clinics $(n=27$ [53\%]), followed by CFAR $(n=$ $14[27 \%])$ and ACTG $(n=10[20 \%])$.

\section{Clinic Type, Screening Access, Personnel}

Thirty-three respondents (65\%) described their site as a clinical research setting and $34(67 \%)$ described their site as an HIV care and treatment clinic. Additional responses included 12 family planning clinics (24\%), 9 antenatal care clinics (18\%), 7 primary care clinics (14\%), and 5 other (10\%; tertiary care hospital, sexually transmitted disease clinic, or cancer center).

Of the 49 sites $(96 \%)$ that had access to cervical cancer screening (see Table 2), 39 (80\%) had screening services on-site. Electronic medical records were used at 17 sites (44\%) with onsite screening. Sites in Central Africa were less likely to have screening on-site compared with the other regions (4 [67\%] vs $8[18 \%] ; p=.02)$. Specifically, none of the Burundi or Rwanda sites reported on-site screening. In East Africa, 5 (71\%) of the 7

\section{TABLE 1. Survey Questions}

1. What type of clinic is your program? (multiple choice: antenatal clinic, clinical research site, family planning, HIV care site, primary care clinic, other; more than one answer may be appropriate)

2. Is cervical cancer screening available to your patients (either on site or via referral)? (Yes, No)

3. If cervical cancer screening is available on site:

Does your program screen women with HIV infection? (Yes, No)

Does your program screen women without HIV infection? (Yes, No)

Does your program maintain electronic records on women screened? (Yes, No)

Cervical cancer screening is performed by: (multiple choice: clinical officer, nurse, lay health worker, physician, other; more than 1 answer may be appropriate)

The method(s) used for cervical cancer screening are: (multiple choice: Pap, VIA, HPV DNA, VILI; more than 1 answer may be appropriate)

Treatments available for premalignant lesions and cervical cancer: (multiple choice: cryotherapy, conization, LEEP, radical hysterectomy, radiation therapy, chemotherapy, other; more than 1 answer may be appropriate)

Patients are referred to our facility for cervical cancer screening (Yes, No)

Patients are referred from: (multiple choice: antenatal clinic, clinical research site, family planning, HIV care site, primary care clinic, other)

4. If cervical cancer screening is available at referral site:

Distance to referral site

Surveys were emailed to the affiliated sites and completed by a site representative. Some questions permitted more than 1 answer.

VIA indicates visual inspection with acetic acid; HPV, human papillomavirus; VILI, visual inspection with Lugol solution; LEEP, loop electrosurgical excision procedure. 


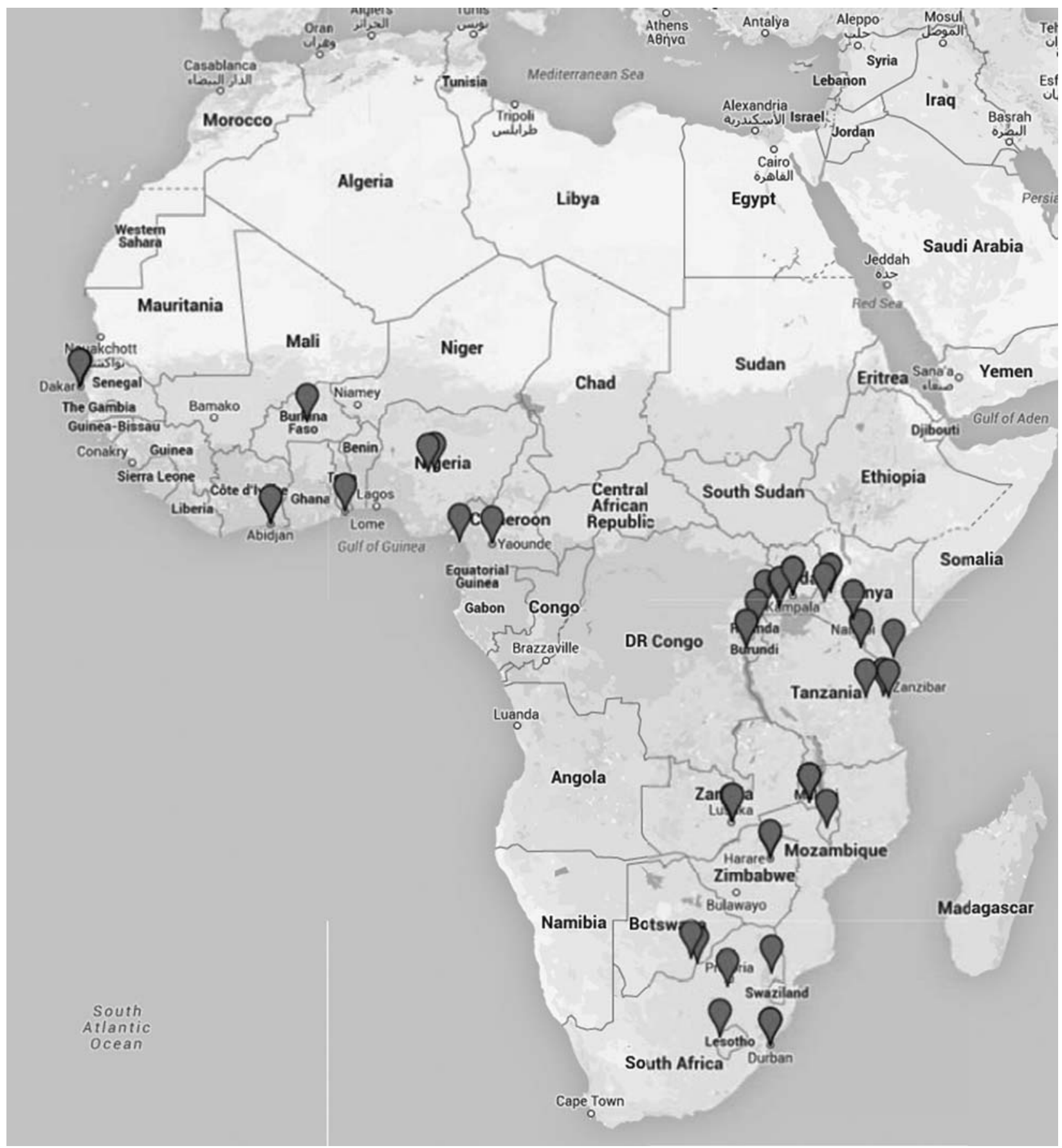

FIGURE 1. Location of Responding Sites.

Ugandan sites did not have on-site screening and 2 of these sites did not have access to screening services elsewhere.

For the 39 sites providing on-site screening, nurses performed screening at 32 sites $(82 \%)$, including 10 sites $(26 \%)$ that relied on nurses exclusively. Physicians provided screening at 25 sites $(64 \%)$, including 6 sites $(24 \%)$ that relied on physicians exclusively. Clinical officers provided screening at 11 sites (28\%), and none of these sites relied on clinical officers exclusively. The remainder of the sites reported a combination of providers, including 1 site that reported the availability of lay health workers.

\section{On-Site Screening and Treatment Modalities}

Visual inspection with acetic acid was the most commonly reported screening method, which was available at 31 sites $(79 \%)$. Eleven $(35 \%)$ of these 31 sites used VIA as the sole screening method. Visual inspection with Lugol solution was reported less commonly at 11 sites (28\%). Pap testing was the second most commonly reported screening method, which was used at 26 sites (67\%). Four (15\%) of these 26 sites used Pap testing as the sole screening method. High-risk HPV testing was available at 13 sites 
TABLE 2. Screening Method by Country

\begin{tabular}{|c|c|c|c|c|c|c|c|c|}
\hline \multirow[b]{2}{*}{ Site (by region and country) } & \multirow[b]{2}{*}{ No. sites } & \multirow{2}{*}{$\begin{array}{l}\text { No. on-site } \\
\text { screening programs }\end{array}$} & \multicolumn{5}{|c|}{ Screening method } & \multirow{2}{*}{$\begin{array}{l}\text { No. sites with electronic } \\
\text { medical records }\end{array}$} \\
\hline & & & Pap & VIA & VILI & HPV & Colpo/bx & \\
\hline \multicolumn{9}{|l|}{ Central Africa $(n=6)$} \\
\hline Burundi & 3 & 0 & $0(0)$ & $0(0)$ & $0(0)$ & $0(0)$ & $0(0)$ & $0(0)$ \\
\hline Cameroon & 2 & 2 & $2(100)$ & $0(0)$ & $0(0)$ & $1(50)$ & $0(0)$ & $0(0)$ \\
\hline Rwanda & 1 & 0 & $0(0)$ & $0(0)$ & $0(0)$ & $0(0)$ & $0(0)$ & $0(0)$ \\
\hline \multicolumn{9}{|l|}{ East Africa $(n=18)$} \\
\hline Kenya & 6 & 6 & $5(83)$ & $6(100)$ & $2(33)$ & $3(50)$ & $0(0)$ & $5(83)$ \\
\hline Tanzania & 5 & 5 & $1(20)$ & $5(100)$ & $1(20)$ & $1(20)$ & $0(0)$ & $0(0)$ \\
\hline Uganda & 7 & 2 & $1(50)$ & $2(100)$ & $1(50)$ & $0(0)$ & $0(0)$ & $0(0)$ \\
\hline \multicolumn{9}{|l|}{ Southern Africa $(n=19)$} \\
\hline Botswana & 2 & 2 & $2(100)$ & $1(50)$ & $1(50)$ & $1(50)$ & $1(50)$ & $1(50)$ \\
\hline Lesotho & 1 & 1 & $1(100)$ & $1(100)$ & $0(0)$ & $0(0)$ & $0(0)$ & $1(100)$ \\
\hline Malawi & 7 & 7 & $3(43)$ & $7(100)$ & $2(29)$ & $2(29)$ & $1(14)$ & $1(14)$ \\
\hline South Africa & 5 & 4 & $4(100)$ & $2(50)$ & $1(25)$ & $2(50)$ & $2(50)$ & $3(75)$ \\
\hline Zambia & 2 & 2 & $0(0)$ & $2(100)$ & $0(0)$ & $0(0)$ & $0(0)$ & $2(100)$ \\
\hline Zimbabwe & 2 & 2 & $1(50)$ & $2(100)$ & $0(0)$ & $0(0)$ & $1(50)$ & $2(100)$ \\
\hline \multicolumn{9}{|l|}{ West Africa $(n=8)$} \\
\hline Burkina Faso & 1 & 1 & $1(100)$ & $1(100)$ & $1(100)$ & $1(100)$ & $0(0)$ & $1(100)$ \\
\hline Côte d'Ivoire & 2 & 1 & $1(100)$ & $1(100)$ & $1(100)$ & $0(0)$ & $0(0)$ & $0(0)$ \\
\hline Nigeria & 2 & 2 & $2(100)$ & $1(50)$ & $1(50)$ & $1(50)$ & $0(0)$ & $1(50)$ \\
\hline Senegal & 2 & 1 & $1(100)$ & $0(0)$ & $0(0)$ & $1(100)$ & $0(0)$ & $0(0)$ \\
\hline Togo & 1 & 1 & $1(100)$ & $0(0)$ & $0(0)$ & $0(0)$ & $0(0)$ & $0(0)$ \\
\hline Total & 51 & 39 & $26(67)$ & 31 (79) & $11(28)$ & $13(33)$ & $5(13)$ & $17(44)$ \\
\hline
\end{tabular}

Data are presented as $n(\%)$.

Denominator is the number of on-site screening programs.

VIA indicates visual inspection with acetic acid; VILI, visual inspection with Lugol solution; HPV, human papilloma virus DNA test; colpo/bx, colposcopy \pm biopsy.

(33\%). Neither VILI nor hrHPV DNA testing was ever used as the sole screening method. Most sites $(62 \%)$ reported the availability of multiple screening methods. High-risk HPV testing was available at $29 \%$ of sites with VIA or VILI and $50 \%$ of sites with Pap testing. Colposcopy was only available at sites that offered Pap testing. Of the 26 sites with Pap testing, 5 (19\%) had colposcopy and 4 (15\%) had colposcopy with biopsy services. Sites with on-site colposcopy and biopsy services were more likely to be located in Southern Africa compared with other regions in SSA (5 $[28 \%]$ vs $0[0 \%], p=.02)$.

Most sites that offered on-site screening also had treatment available $(n=36$ [92\%]) and some had multiple treatment options available. Cryotherapy was the most commonly reported method available to treat premalignant lesions, which was used at 29 sites (74\%). Excisional procedures, such as a loop electrosurgical excision procedure (LEEP) and cold-knife cone biopsy, were offered at $23(59 \%)$ and $12(31 \%)$ sites, respectively. Of the sites that offered cryotherapy, $24(77 \%)$ reported having either LEEP or cold-knife cone biopsy available, if needed. Radical or extended hysterectomy was the most commonly reported method available to treat ICC, which was used at 18 sites $(46 \%)$. Fewer sites had chemotherapy or radiation therapy at $10(26 \%)$ and $6(15 \%)$, respectively. Of the sites that offered radical or extended hysterectomy, $9(50 \%)$ had chemotherapy, $5(28 \%)$ had radiation therapy, and $4(22 \%)$ had both chemotherapy and radiation available.

Nearly 37 sites (95\%) reported on-site cervical cancer prevention programs for women with HIV infection, and $8(22 \%)$ of these sites restricted services to women with HIV infection alone (see Table 3). There were no significant differences between the screening and treatment modalities available at the sites that offered services to women with HIV infection compared with those that offered services to both HIV infected and uninfected (data not shown).

\section{Referral Center Designation and Access}

Thirty sites (59\%) served as a referral center for cervical cancer screening. Of these, $26(87 \%)$ received referrals from other HIV care and treatment programs and $20(67 \%)$ received referrals from primary care clinics. The remainder of the referrals to the individual programs originated from family planning clinics 17 (60\%), clinical research sites $17(57 \%)$, antenatal care clinics 15 $(50 \%)$, and other clinic types $8(27 \%)$. Sites were queried on the access to referral centers for cervical cancer screening that was not available at their clinic. For the 12 sites $(24 \%)$ that did not have on-site screening, $10(83 \%)$ of their referral sites were located within the same facility or less than $10 \mathrm{~km}$ away, and 2 sites $(17 \%)$ did not have access to a referral clinic.

\section{DISCUSSION}

Most sites affiliated with an NIH-funded HIV/AIDS network in our study had on-site cervical cancer screening programs, and almost all had access to screening, even if screening was not available on-site. Some of these sites also served as referral centers from other treatment programs. These data show that the infrastructure for cervical cancer screening and linkages between clinics have been established in many urban areas; however, there remain a few challenges. 
TABLE 3. Sites With On-Site Screening and Treatment Available by HIV Status ${ }^{a, b}$

\begin{tabular}{lcr}
\hline & $\begin{array}{c}\text { HIV infected } \\
(\boldsymbol{n}=\mathbf{3 7})\end{array}$ & $\begin{array}{c}\text { HIV uninfected } \\
(\mathbf{n}=\mathbf{2 9})\end{array}$ \\
\hline Screening & $29(78)$ & $24(83)$ \\
VIA & $25(68)$ & $19(66)$ \\
Pap & $13(35)$ & $10(34)$ \\
HPV DNA & $10(27)$ & $9(31)$ \\
VILI & $4(11)$ & $3(10)$ \\
Colposcopy \pm biopsy & & \\
Treatment & $3(8)$ & $1(3)$ \\
$\quad$ None & & \\
Premalignant lesions & $27(73)$ & $21(72)$ \\
Cryotherapy & $21(57)$ & $17(59)$ \\
LEEP & $12(32)$ & $10(34)$ \\
Conization & & \\
Malignant lesions & $18(49)$ & $16(55)$ \\
Radical or extended hysterectomy & $9(24)$ & $10(34)$ \\
Chemotherapy & $5(14)$ & $5(17)$ \\
$\quad$ Radiation therapy &
\end{tabular}

Data are presented as $\mathrm{n}(\%)$

VIA indicates visual inspection with acetic acid; VILI, visual inspection with Lugol solution; HPV, human papilloma virus DNA test; LEEP, loop electrosurgical excision procedure.

${ }^{a}$ Sites may report more than 1 screening and treatment method.

${ }^{b}$ Sites may be included in both women with HIV infection and without HIV infection columns if sites report screening and treatment programs regardless of HIV status.

Most sites with several cervical cancer screening methods available performed VIA; however, many sites continue to offer Pap testing. Ideally, abnormal Pap tests are managed using colposcopy and biopsy, if needed, but most of these sites did not have an available colposcope or pathology services. A recent quality assurance program for cervical cytology and histology showed that an education program for pathologists in resource-constrained settings can lead to improved diagnostic interpretations. ${ }^{17}$ However, studies have shown that there is a shortage of pathologists in SSA, which contributes to the long interval between biopsy results and treatment. ${ }^{18}$ Scale-up of Pap testing for many millions of women would likely create a large burden on the scant pathology services in much of SSA. In addition, the complexity of Pap testing programs may increase the proportion of patients who do not return for treatment because multiple visits are usually necessary, which requires reliable communication with and transportation for patients. Khozaim et al. ${ }^{19}$ reported that loss to follow-up is one of the major challenges of cervical cancer prevention programs because a third of their patients in Western Kenyan did not return for treatment. Currently, a better approach is the single-visit "screen-and-treat" strategy that uses visual inspection techniques, followed by cryotherapy for eligible women. However, future availability of lower-cost, point-of-care rapid hrHPV DNA testing should also be incorporated into the screen-and-treat strategy to improve specificity and better identify the women with premalignant lesions who could benefit most from immediate treatment. ${ }^{20}$ High-risk HPV testing has not been widely available to date, but a new point-of-care cartridge-based molecular diagnostic system for hrHPV is now available in SSA that requires very little laboratory infrastructure and provides results in 1 hour allowing for same-day test-and-treat approaches. ${ }^{21}$ These single-visit approaches decrease barriers to care, are affordable, and are sustainable, as opposed to Pap testing and colposcopy. ${ }^{9-11,22-24}$

In addition to screening, a successful cervical cancer prevention program requires treatment of premalignant lesions. Cryotherapy has been shown to be an effective treatment modality, regardless of HIV status, but has higher subsequent disease negative rates among women without HIV infection. ${ }^{25}$ In women with HIV infection, premalignant lesions tend to be larger and involve the endocervical canal, which makes it more likely that an additional treatment method, for example, LEEP or cold-knife cone biopsy, is needed. ${ }^{6,26}$ Although we found that most sites that offered treatment for premalignant lesions had cryotherapy, almost a quarter of these sites did not have excisional procedures such as LEEP available although the sites provided care to women with HIV infection. Cervical cancer prevention sites should increase access to excisional procedures, either on-site or by referral, which will involve additional training for providers and implementation of safety and quality control measures.

Integral to cervical cancer screening programs are the skilled providers. Our survey showed that physicians performed screening at a large number of sites. However, it has been shown that nurses can successfully perform these tasks. In Zambia, which reportedly has one of the highest mortality rates from cervical cancer worldwide, 1 program employs trained nurses to provide digital cervicography-aided VIA with same-day cryotherapy. ${ }^{27}$ Digital images of suspicious lesions are reviewed in real time by gynecologists at remote tertiary care sites. Even among women with HIV infection, the sensitivity of cervicography-aided VIA to detect CIN 2/3 lesions was higher than Pap testing. ${ }^{28}$ Thus, task shifting from physicians- to nurse-led programs can lower costs and expand access without compromising effectiveness.

Furthermore, a successful cervical cancer prevention program requires treatment of malignant lesions; however, the management of ICC is a major challenge in SSA countries because of the lack of access to and varied quality of cancer treatment centers. In our survey, fewer than half of sites were able to perform radical or extended hysterectomies and an overwhelming majority did not have chemotherapy or radiation therapy. An even smaller proportion had all 3 options available. A 2008 review on cancer treatment in SSA noted that radiotherapy was available in only 23 of 53 countries and that although there was a $30 \%$ increase in sites during the previous decade, this expansion occurred in countries already able to offer radiotherapy and was not expanded to countries that did not have any access to radiation. ${ }^{29}$ To decrease ICC deaths, adequate and appropriate treatment options must be expanded, with special attention to those countries that did not have any access previously.

Our study has limitations. First, there may have been sampling bias. The survey was sent only to NIH-funded HIV/AIDS sites, which may have impacted the responses. For example, the high proportion of sites that offered Pap testing might be because US investigators, who are accustomed to using the Pap test as the primary screening method, led these sites. In addition, more than a third of sites that were invited to participate in the study did not respond. Besides geographic location, we do not have any additional details about these nonresponding sites. It is possible that sites without cervical cancer screening did not respond, which would decrease our reported percentages of sites with on-site screening availability. Second, we did not query Ministries of Health, the private sector, or other organizations that might have robust cervical cancer prevention programs. Therefore, our results may not be indicative of the country's response to cervical cancer screening and may not reflect the general environment. However, more than half of our respondents were a part of the IeDEA network, which collects data from routine HIV clinical visits and thus provides some 
insight into services offered at local healthcare facilities. Next, there might be misclassification. For example, on-site screening services might have been interpreted literally, meaning it is possible that a site could have reported not having on-site screening services; however, another building on-campus might have screening services. Last, SSA is undergoing scale-up of cervical cancer screening services and our results represent status through the end of 2013.

Overall, the higher incidence and mortality of cervical cancer in developing countries can be attributed to a generalized lack of awareness, absence or poor quality of screening programs, and poor access to care, prevention and treatment. Building upon the existing infrastructure in established care delivery systems in resource-constrained settings represents an ideal framework for implementing a program to reduce cervical cancer incidence. Although we surveyed HIV programs, it is important to note that routine cervical cancer screening among the general population in many countries typically was introduced by HIV clinics. Cost containment and skilled staff who are able to perform screening and treatment are integral to the sustainability of a cervical cancer prevention program. It is possible that a coordinated network can be established that could serve as a platform to accelerate the implementation of evidence-based cancer prevention programs for women with HIV infection and without HIV infection. There should be a collective effort among public, private, and academic sectors to increase screening and treatment programs, determine the most effective screening algorithm, particularly for women with HIV infection, and conduct cancer research to halt the projected rise of ICC in resource-constrained settings.

\section{ACKNOWLEDGMENTS}

We are grateful to R. Scott McClelland and Mirjam-Colette Kempf for their critical reading of the manuscript.

We would like to thank the following countries, institutions, clinical site investigators, and staff who participated in the survey, in alphabetical order: Botswana: Botswana Harvard HIV Reference Lab, Gaborone and Molepolole CRS; Burkina Faso: Hospital du Jour (CHU-Yalgado Ouedraogo) Ouagadougou; Burundi: Association Nationale de soutien aux Seropositifs et Malades du Sida (ANSS), Centre Hospitalo-Universitaire de Kamenge (CHUK), and Hôspital Prince Régent Charles (HPRC CPAMP); Cameroon: Regional Hospital Limbe, Yaounde General Hospital; Côte d'Ivoire: KO'KHOUA, CNTS/CMSDS (Centre Medical de Suivi des Donneurs de Sang), CIRBA (Centre Intégré de Recherche Bioclinique d'Abidjan) and USAC (UNITE DE SOINS AMBULATOIRES ET DE CONSEILS) Abidjan; Kenya: AMPATH Cervical Cancer Screening Program at Moi Teaching and Referral Hospital, Coptic Hope Center for Infectious Diseases, University of Nairobi/University of Washington Mombasa HIV and STD Research Field Site, Family AIDS Care and Education services and AMPATH Reference Lab Moi University; Lesotho: Senkatana Cervical Screening Center of Excellence; Malawi: Bwaila Family Health Unit Clinics, Kamuzu Central Hospital Colposcopy Clinic, Kamuzu Central Hospital STI Clinic, UNC Project-Malawi Clinics, Partners in Hope, Lighthouse Trust Clinics, and College of Medicine JHU CRS; Nigeria: NHA (National Hospital Abuja) and UATH (University of Abuja Teaching Hospital; Gwagwalada) Abuja; Rwanda: Rwanda Military Hospital; Senegal: SMIT/ CRCF (Service de Maladies Infectieuses et Tropicales/Centre Régional de Recherche et de Formation à la Prise en Charge Clinique de Fann) (Dakar, Sénégal), CHNU de Fann, Dakar; South Africa: Themba Lethu Clinic at Helen Joseph Hospital, uBuntu, Durban Adult HIV CRS, CLS University of Witwatersrand, and CAPRISA; Tanzania: KCMC Women's Health Program, Morogoro Regional Hospital, Ocean Road Cancer Institute, Tumbi Regional
Hospital, and KCMC-Moshi; Togo: SMIT (Service de Maladies Infectieuses et Tropicales)/CHU SYLVANUS Olympio, Lome; Uganda: Makerere Palliative Care Unit Department of Medicine at Mulago Hospital, Mulago Hospital-Department of OB-GYN, Mulago Hospital-Uganda Cancer Institute, Infectious Diseases Institute (IDI), Masaka Regional Referral Hospital HIV Clinic, Mbarara University of Science and Technology, ISS Clinic, and Rakai Health Sciences Program; Zambia: Centre for Infectious Disease Research in Zambia and Kalingalinga Clinic CRS, Zimbabwe: Newlands Clinic and UZ-Parirenya TWA CRS.

\section{REFERENCES}

1. Ferlay J, Soeriomataram I, Ervik M, et al. Estimated Cancer Incidence, Mortality, and Prevalence Worldwide in 2012. Lyon, France: Cancer IAfRo; 2012

2. World Health Organization. Comprehensive Cervical Cancer Prevention and Control: A Healthier Future for Girls and Women. Switzerland; 2013.

3. Jemal A, Bray F, Forman D, et al. Cancer burden in Africa and opportunities for prevention. Cancer 2012;118:4372-84.

4. National Cancer Institute. Cervical Cancer. Health NIo; 2014.

5. Firnhaber C, Zungu K, Levin S, et al. Diverse and high prevalence of human papillomavirus associated with a significant high rate of cervical dysplasia in human immunodeficiency virus-infected women in Johannesburg, South Africa. Acta Cytol 2009;53:10-7.

6. Maiman M, Fruchter RG, Sedlis A, et al. Prevalence, risk factors, and accuracy of cytologic screening for cervical intraepithelial neoplasia in women with the human immunodeficiency virus. Gynecol Oncol 1998;68:233-9.

7. Uberti-Foppa C, Origoni M, Maillard M, et al. Evaluation of the detection of human papillomavirus genotypes in cervical specimens by hybrid capture as screening for precancerous lesions in HIV-positive women J Med Virol 1998;56:133-7

8. World Health Organization. Global Health Observatory Data Repository: Number of people (all ages) living with HIV Switzerland; 2014.

9. De Vuyst H, Claeys P, Njiru S, et al. Comparison of Pap smear, visual inspection with acetic acid, human papillomavirus DNA-PCR testing and cervicography. Int J Gynaecol Obstet 2005;89:120-6.

10. Sarian LO, Derchain SF, Naud P, et al. Evaluation of visual inspection with acetic acid (VIA), Lugol's iodine (VILI), cervical cytology and HPV testing as cervical screening tools in Latin America. This report refers to partial results from the LAMS (Latin AMerican Screening) study. J Med Screen 2005; 12:142-9.

11. Syrjanen K, Derchain S, Roteli-Martins C, et al. Value of conventional Pap smear, liquid-based cytology, visual inspection and human papillomavirus testing as optional screening tools among latin american women $<35$ and $>$ or $=35$ years of age: experience from the Latin American Screening Study. Acta Cytol 2008;52:641-53.

12. Mabeya H, Khozaim K, Liu T, et al. Comparison of conventional cervical cytology versus visual inspection with acetic acid among human immunodeficiency virus-infected women in Western Kenya. J Low Genit Tract Dis 2012;16:92-7.

13. Sankaranarayanan R, Esmy PO, Rajkumar R, et al. Effect of visual screening on cervical cancer incidence and mortality in Tamil Nadu, India: a cluster-randomised trial. Lancet 2007;370:398-406.

14. Sankaranarayanan R, Nene BM, Shastri SS, et al. HPV screening for cervical cancer in rural India. N Engl J Med 2009;360:1385-94.

15. World Health Organization. WHO Guidelines: WHO Guidelines for Screening and Treatment of Precancerous Lesions for Cervical Cancer Prevention. Geneva, Switzerland: World Health Organization; 2013.

16. Sylla BS, Wild CP. A million africans a year dying from cancer by 2030 : what can cancer research and control offer to the continent? Int J Cancer 2012;130:245-50. 
17. Godfrey CC, Michelow PM, Godard M, et al. Improving diagnostic capability for HPV disease internationally within the NIH-NIAID Division of AIDS Clinical Trial Networks. Am J Clin Pathol 2013;140: 881-9.

18. Rambau PF. Pathology practice in a resource-poor setting: Mwanza, Tanzania. Arch Pathol Lab Med 2011;135:191-3.

19. Khozaim K, Orang'o E, Christoffersen-Deb A, et al. Successes and challenges of establishing a cervical cancer screening and treatment program in western Kenya. Int J Gynaecol Obstet 2014;124:12-8.

20. Qiao YL, Jeronimo J, Zhao FH, et al. Lower cost strategies for triage of human papillomavirus DNA-positive women. Int J Cancer 2014;134: 2891-901.

21. Einstein MH, Smith KM, Davis TE, et al. Clinical evaluation of the cartridge-based GeneXpert human papillomavirus assay in women referred for colposcopy. J Clin Microbiol 2014;52:2089-95.

22. Joshi S, Sankaranarayanan R, Muwonge R, et al. Screening of cervical neoplasia in HIV-infected women in India. AIDS 2013;27:607-15.

23. Wright TC Jr, Denny L, Kuhn L, et al. Use of visual screening methods for cervical cancer screening. Obstet Gynecol Clin North Am 2002;29: $701-34$.
24. Zhang HY, Tiggelaar SM, Sahasrabuddhe VV, et al. HPV prevalence and cervical intraepithelial neoplasia among HIV-infected women in Yunnan Province, China: a pilot study. Asian Pac J Cancer Prev 2012;13:91-6.

25. Chumworathayi B, Srisupundit S, Lumbiganon P, et al. One-year follow-up of single-visit approach to cervical cancer prevention based on visual inspection with acetic acid wash and immediate cryotherapy in rural Thailand. Int J Gynecol Cancer 2008;18:736-42.

26. Horo A, Jaquet A, Ekouevi DK, et al. Cervical cancer screening by visual inspection in Cote d'Ivoire, operational and clinical aspects according to HIV status. BMC Public Health 2012;12:237.

27. Mwanahamuntu MH, Sahasrabuddhe VV, Blevins M, et al. Utilization of cervical cancer screening services and trends in screening positivity rates in a 'screen-and-treat' program integrated with HIV/AIDS care in Zambia. PLoS One 2013;8:e74607.

28. Bateman AC, Parham GP, Sahasrabuddhe VV, et al. Clinical performance of digital cervicography and cytology for cervical cancer screening in HIV-infected women in Lusaka, Zambia. J Acquir Immune Defic Syndr 2014;67:212-5.

29. Sitas F, Parkin DM, Chirenje M, et al. Part II: cancer in Indigenous Africans - causes and control. Lancet Oncol 2008;9:786-95.

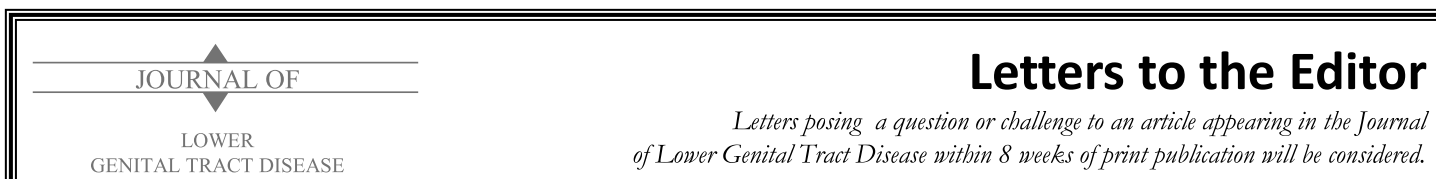

The Editor may send the letter to the authors of the original article so that their response may be published simultaneously.

Following are the format and submission guidelines:

- Limit the letter to a maximum of 350 words, including signature and references. References should not exceed 5 and must include the article to which you are responding. Provide a word count.

- On the first page of your letter, list the title and the names of the authors of the article to which you are responding.

- A maximum of 3 authors is permitted.

- Designate a corresponding author and provide address, telephone numbers, and Email address.

- Submit the text and author agreement form at: http://JLGTD.editorialmanager.com

The Journal of Lower Genital Tract Disease, is sponsored by the American Society for Colposcopy and Cervical Pathology, the International Society for the Study of Vulvovaginal Disease, the Australian Society for Colposcopy and Cervical Pathology, the Society of Canadian Colposcopists, and the International Federation of Cervical Pathology and Colposcopy. The Journal is dedicated to the study and interchange of ideas regarding health and diseases of the lower genital tract. The Editor reserves the right to shorten letters, delete irrelevant or objectionable commentary, and make changes to comply with journal style. An effort is made to publish letters within two months of acceptance. All letters will be published on the journal E-pages. 